\title{
A Dynamic Anti-windup AQM for Congestion Control in Internet
}

\author{
Nabil EL FEZAZI ${ }^{1}$, Sadek BELAMFEDEL ALAOUI ${ }^{1}$, Fatima EL HAOUSSI ${ }^{1,2}$, \\ El Houssaine TISSIR $^{1}$ and Teresa ALVAREZ ${ }^{3}$
}

\begin{abstract}
This paper addresses the design of anti-windup gains for obtaining stability for linear systems with discrete time varying delay and saturating inputs delay. Considering that a dynamic output feedback has been designed to stabilize the AQM system (without saturation), a method is proposed for designing an anti-windup gain which relates the saturation of the queue when congestion, which is inevitable in networks. It is shown that the closed-loop system obtained from the controller plus the anti-windup gain can be modeled by a linear system with a dead zone nonlinearity. A modified sector condition is then used to obtain stability conditions based on Lyapunov functions. Differently from previous works these conditions are directly in linear matrix inequality form. Resulting in a new AQM which will be simulated in MATLAB and compared to RED.
\end{abstract}

\section{INTRODUCTION}

Active queue management (AQM) is an effective solution for the congestion control problem. It can achieve high quality of service (QoS) by reducing the packet dropping probability and improving network utilization. It is implemented in router to inform TCP senders about a current congestion. Based on this information senders adapts their sending rate to the state of network. A lot of Active queue management are developed in the literature, among them, Random Early Detection (RED): monitors the average queue size and drops (or marks when used in conjunction with ECN) packets based on statistical probabilities, and PI controller (proportionalintegral controller) is a special case of the PID controller in which the derivative (D) of the error is not used [18], [1], [2]. These controllers are widely used in practice, because their implementation is simple. On the other hand, many automatic controllers use artificial intelligence (fuzzy logic, neural networks, ...) [3], [6], [11], [17], [18]. These controllers give very good results, but the implementation of their algorithms requires the presence of an expert. Note that the works above do not consider plant input saturation. Since the control action in AQM is determining the discarting probability, which is clearly a real number bounded between $[0,1]$, you cannot have a realistic AQM that disregards input saturation. In order to mitigate the saturation effect on stability of systems, an anti-windup synthesis for state-delayed systems has been addressed in [5], [7], [8], [9], [10], [16], [19]. It must

\footnotetext{
${ }^{1}$ Sidi Mohammed Ben Abdellah University, Department of Physics, Faculty of Sciences Dhar El Mehraz, LESSI, BP 1796, Fes-Atlas, Morocco. fizazi.99@gmail.com, fedelkds@gmail.com, elhoussaine.tissir@usmba.ac.ma

${ }^{2}$ Polydisciplinary Faculty, BP 300, Selouane 62700, Nador, Morocco. elhaous_fati@yahoo.fr

${ }^{3}$ Valladolid University, Department of Systems Engineering and Automation, 47005, Valladolid, Spain. tere@autom.uva.es
}

be noted that these works neglect the system discretizing which is very important to study the real system. Thus, we extend the output dynamic used in [9] and [10] to this work. The dynamics of this controller has been chosen so that the closed loop system is stable. The anti-windup compensator itself emits two signals, one which is fed directly into the constrained control signal and one which may be used to drive the controller state equation directly. Virtually all antiwindup compensators which are present in the literature can be represented in the form of [16] and the anti-windup compensator discussed here will be the same type.

The objective of this paper is to design a controller which is capable of achieving the queue size and guaranteeing the stability of saturated discrete TCP/AQM systems with both link capacity disturbances. For this reason, dynamic antiwindup AQM control is very simple to implement and give good results. To explain the controller design, we organized this paper as follows: a problem formulation under study and useful lemmas are presented in Section II. We announce a main theorem and this proof, an optimization problem and an important implementation of our AQM are discussed in Section III. Finally, a numerical example is included to illustrate the developed results.

\section{PRoblem Formulation AND PRELIMINARIES}

Our study will focus on the sharing of a communication link between multiple transmitters at remote locations. We consider a single bottleneck router running TCP flows as illustrated in the following figure

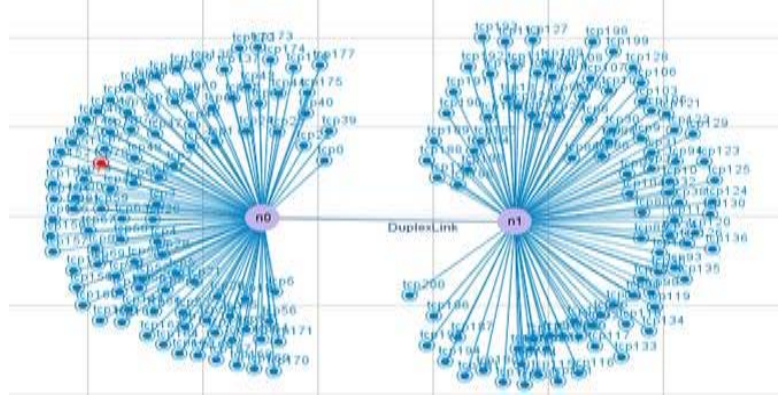

Fig. 1. Simulation of network topology

The model of TCP behavior relating the average value of key network variables is described by the following coupled equations [13], [14]

$$
\begin{aligned}
\dot{W}(t) & =\frac{1}{R T T(t)}-\frac{W(t) W(t-R T T(t))}{2 R T T(t-R T T(t))} p(t-R T T(t)) \\
\dot{q}(t) & =-C(t)+\frac{N(t)}{R T T(t)} W(t)
\end{aligned}
$$


where

$W(t)$ is the average TCP window size (packets);

$q(t)$ is the average queue length (packets);

$R T T(t)$ is the round trip time $=\frac{q(t)}{C(t)}+T_{p}(\operatorname{secs})$;

$C$ is the link capacity (packets/secs);

$T_{p}$ is the propagation delay (secs);

$N$ is the number of sessions;

$p \in\left[\begin{array}{ll}0 & 1\end{array}\right]$ is the probability of packet marking/dropping.

The window size and the queue length are positive and bounded; i.e. $W \in\left[\begin{array}{ll}0 & W_{\max }\end{array}\right]$ and $q \in\left[\begin{array}{ll}0 & q_{\max }\end{array}\right]$. For a given triplet of network parameters $\left(N, C_{0}, T_{p}\right)$, any triplet $\mathfrak{I}=\left(W_{0}, q_{0}, p_{0}\right)$, let

$$
\Xi=\left\{\mathfrak{I}: W_{0}=\frac{R T T C_{0}}{N}, q_{0}=C_{0}\left(R T T-T_{p}\right), p_{0}=\frac{2}{W_{0}^{2}}\right\}
$$

be a possible operating point. Now define $\delta \mathfrak{C}=\mathfrak{C}-\mathfrak{C}_{0}$ with $\mathfrak{C}=W, q, p, C$. Then, we can obtain the linearized version of (1) as follows

$$
\begin{aligned}
\delta \dot{W}(t)= & \frac{-N}{R T T^{2} C_{0}}(\delta W(t)+\delta W(t-R T T(t))) \\
& -\frac{1}{R T T^{2} C_{0}}(\delta q(t)+\delta q(t-R T T(t))) \\
& -\frac{R T T C_{0}^{2}}{2 N^{2}} \delta p(t-R T T(t)) \\
& +\frac{R T T-T_{p}}{R T T^{2} C_{0}}(\delta C(t)+\delta C(t-R T T(t))) \\
\delta \dot{q}(t)= & \frac{N}{R T T} \delta W(t)-\frac{1}{R T T} \delta q(t)-\frac{T_{p}}{R T T} \delta C(t) \\
R T T(t)= & \frac{\delta q(t)}{C_{0}}+R T T
\end{aligned}
$$

Thus, rewriting (2) in state space form yields

$$
\begin{aligned}
\dot{x}(t) & =A x(t)+A_{\tau} x(t-\tau(t))+B u(t-\tau(t))+B_{w} w(t) \\
y(t) & =C_{y} x(t) \\
z(t) & =C_{z} x(t)
\end{aligned}
$$

in which

$$
\begin{aligned}
& x(t)=\left[\begin{array}{c}
\delta W(t) \\
\delta q(t)
\end{array}\right], w(t)=\left[\begin{array}{c}
\delta C(t) \\
\delta C(t-R T T(t))
\end{array}\right], \\
& u(t)=\delta p(t), y(t)=\delta q(t), z(t)=R T T(t)-R T T, \\
& A=\left[\begin{array}{cc}
\frac{-N}{R T T^{2} C_{0}} & \frac{-1}{R T T^{2} C_{0}} \\
\frac{N}{R T T} & \frac{-1}{R T T}
\end{array}\right], A_{d}=\left[\begin{array}{cc}
\frac{-N}{R T T^{2} C_{0}} & \frac{-1}{R T T^{2} C_{0}} \\
0 & 0
\end{array}\right], \\
& B=\left[\begin{array}{cc}
\frac{-R T T C_{0}^{2}}{2 N^{2}} \\
0
\end{array}\right], B_{w}=\left[\begin{array}{cc}
\frac{R T T-T_{p}}{R T T^{2} C_{0}} & \frac{R T T-T_{p}}{R T T^{2} C_{0}} \\
\frac{-T_{p}}{R T T} & 0
\end{array}\right], \\
& C_{y}=\left[\begin{array}{ll}
0 & 1
\end{array}\right], C_{z}=\left[\begin{array}{ll}
0 & \frac{1}{C_{0}}
\end{array}\right] .
\end{aligned}
$$

The discretization of the system (3) gives

$$
\begin{aligned}
x(k+1)= & A_{d} x(k)+A_{\tau_{d}} x(k-d(k))+B_{d} u(k-d(k)) \\
& +B_{w_{d}} w(k) \\
y(k)= & C_{y_{d}} x(k) \\
z(k)= & C_{z_{d}} x(k)
\end{aligned}
$$

where $A_{d}=e^{A T}, A_{\tau_{d}}=e^{A_{\tau} T}, C_{y_{d}}=C_{y}, C_{z_{d}}=C_{z}, B_{d}=$ $\int_{0}^{T} e^{A_{\tau}(T-s)} B d s, B_{w_{d}}=e^{B_{w} T}$ and $d(k)$ is a positive integer representing the time delay of the system that we assume to be time dependent and to satisfy the following

$$
d_{m} \leq d(k) \leq d_{M}
$$

where $d_{m}$ and $d_{M}$ are known positive and finite integers.

Then, we have

$$
\begin{aligned}
& A_{d}=\left[\begin{array}{cc}
1-e^{\frac{-T}{R T T^{2} C_{0}}} & e^{\frac{-N T}{R T T^{2} C_{0}}} \\
e^{\frac{-T}{R T T}} & \lambda
\end{array}\right], A_{\tau_{d}}=\left[\begin{array}{cc}
e^{\frac{-T}{R T T}} & \lambda \\
0 & 0
\end{array}\right], \\
& B_{d}=\left[\begin{array}{c}
1-e^{\frac{-R T T C_{0} T}{N^{2}}} \\
0
\end{array}\right], B_{w_{d}}=\left[\begin{array}{cc}
1-e^{\frac{-T_{p} T}{R T T}} & \beta \\
0 & 0
\end{array}\right]
\end{aligned}
$$

where $\lambda=\frac{N C_{0} R T T}{R T T^{2} C_{0}-2 N}\left(e^{\frac{-2 N T}{R T T^{2} C_{0}}}-e^{\frac{-T}{R T T}}\right) e^{\frac{-N T}{R T T^{2} C_{0}}}$ and $\beta=$ $e^{\frac{-\left(R T T-T_{p}\right) T}{R T T^{2} C_{0}}}$.

Thus, the dynamic output stabilizing controller is considered as

$$
\begin{aligned}
x_{c}(k+1) & =A_{c} x_{c}(k)+B_{c} y(k) \\
y_{c}(k) & =C_{c} x_{c}(k)+D_{c} y(k)
\end{aligned}
$$

The interconnection of this controller with (4) is given by

$$
\begin{aligned}
x(k+1)= & A_{d} x(k)+A_{\tau_{d}} x(k-d(k))+B_{d} \operatorname{sat}\left(y_{c}(k-d(k))\right) \\
& +B_{w_{d}} w(k) \\
y(k)= & C_{y_{d}} x(k) \\
z(k)= & C_{z_{d}} x(k) \\
x_{c}(k+1)= & A_{c} x_{c}(k)+B_{c} y(k)-E_{c} \psi\left(y_{c}(k-d(k))\right) \\
y_{c}(k)= & C_{c} x_{c}(k)+D_{c} y(k)
\end{aligned}
$$

The term $E_{c} \psi\left(y_{c}(k-d(k))\right)$ is injected to mitigate the effect of windup caused by saturation and

$$
\psi\left(y_{c}(k-d(k))\right)=y_{c}(k-d(k))-\operatorname{sat}\left(y_{c}(k-d(k))\right)
$$

Note that, $\psi\left(y_{c}(k-d(k))\right)$ corresponds to a decentralized dead-zone nonlinearity.

In this case, the augmented system can be represented as follows

$$
\begin{aligned}
\xi(k+1)= & \mathbb{A} \xi(k)+\mathbb{A}_{d} \xi(k-d(k))-\left(\mathbb{B}+\mathbb{R} E_{c}\right) \\
& \times \psi(\mathbb{K} \xi(k-d(k)))+\mathbb{B}_{w} w(k) \\
z(k)= & \mathbb{C}_{z} \xi(k)
\end{aligned}
$$

and the augmented state and matrices are given by

$$
\begin{aligned}
& \xi(k)=\left[\begin{array}{c}
x(k) \\
x_{c}(k)
\end{array}\right], \mathbb{A}=\left[\begin{array}{cc}
A_{d} & 0 \\
B_{c} C_{y_{d}} & A_{c}
\end{array}\right], \mathbb{B}=\left[\begin{array}{c}
B_{d} \\
0
\end{array}\right], \\
& \mathbb{A}_{d}=\left[\begin{array}{cc}
A_{\tau_{d}}+B_{d} D_{c} C_{y_{d}} & B_{d} C_{c} \\
0 & 0
\end{array}\right], \mathbb{B}_{w}=\left[\begin{array}{c}
B_{w_{d}} \\
0
\end{array}\right], \\
& \mathbb{R}=\left[\begin{array}{c}
0 \\
I_{n_{c}}
\end{array}\right], \mathbb{K}=\left[\begin{array}{ll}
D_{c} C_{y_{d}} & C_{c}
\end{array}\right], \mathbb{C}_{z}=\left[\begin{array}{ll}
C_{z_{d}} & 0
\end{array}\right] .
\end{aligned}
$$

Denote by $\mathfrak{F}_{z w}$ the closed-loop transfer function from $w(k)$ to $z(k)$. The objective of the $H_{\infty}$ control design is to find a controller such that

$$
\left\|\mathfrak{F}_{z w}\right\|_{2}<\sqrt{\gamma}
$$


and to minimize $\gamma$ if possible. Clearly, $\gamma$ describes a kind of disturbance rejection ratio between the controlled variable and the exogenous disturbance.

Furthermore, consider a matrix $G \in \mathfrak{R}^{m \times n}$ and define the following polyhedral set

$$
\mathscr{S}=\left\{\xi(k) \in \mathfrak{R}^{n} ;\left|\left(\mathbb{K}_{(k)}-G_{(k)}\right) \xi(k)\right| \leq u_{0_{(k)}}\right\}
$$

the following useful lemmas will be used in this paper

Lemma 2.1: [7] If $\xi(k) \in \mathscr{S}$, then the following relation

$$
\psi^{T}(\mathbb{K} \xi(k)) T[\psi(\mathbb{K} \xi(k))-G \xi(k)] \leq 0
$$

is verified for any diagonal positive matrix $T \in \mathfrak{R}^{m \times m}$.

\section{MAIN RESULTS}

\section{A. Stability Results}

In congestion control, one important problem is to find maximum allowable upper bound of the time delay such that the network can still be stabilized or find the $H_{\infty}$ performance index can still be guaranteed. This problem can be easily dealt based on the following Theorem.

Theorem 3.1: If there exist symmetric positive definite matrices $\widehat{P}, \widehat{Q}, \widehat{R}$, appropriately sized matrices $F_{c}, \widehat{T}, \widehat{G}, \widehat{Y}_{1}, \widehat{Y}_{2}$, $\widehat{Y}_{3}, \widehat{Y}_{4}, \widehat{Y}_{5}$ and positive scalar $\gamma$ satisfying the LMI (11)

$$
\left[\begin{array}{ccccccc}
\Pi_{11} & \Pi_{12} & \Pi_{13} & \Pi_{14} & \Pi_{15} & \Pi_{16} & \Pi_{17} \\
* & \Pi_{22} & \Pi_{23} & \Pi_{24} & \Pi_{25} & \Pi_{26} & 0 \\
* & * & \Pi_{33} & \Pi_{34} & \Pi_{35} & 0 & 0 \\
* & * & * & \Pi_{44} & 0 & \Pi_{46} & 0 \\
* & * & * & * & \Pi_{55} & \Pi_{56} & 0 \\
* & * & * & * & * & \Pi_{66} & 0 \\
* & * & * & * & * & * & \Pi_{77}
\end{array}\right]<0
$$

where $d_{M m}=d_{M}-d_{m}$ and

$$
\begin{aligned}
& \Pi_{11}=-\widehat{P}+\widehat{Q}+d_{M m} \widehat{R}-\widehat{Y}_{1}-\widehat{Y}_{1}^{T}, \Pi_{12}=\widehat{Y}_{1}-\widehat{Y}_{2}^{T} \\
& \Pi_{22}=-\widehat{Q}+\widehat{Y}_{2}+\widehat{Y}_{2}^{T}, \Pi_{13}=\widehat{Y}_{1}-\widehat{Y}_{3}^{T}, \Pi_{23}=\widehat{Y}_{2}+\widehat{Y}_{3}^{T} \\
& \Pi_{33}=\widehat{Y}_{3}+\widehat{Y}_{3}^{T}, \Pi_{14}=-\widehat{Y}_{4}^{T}, \Pi_{24}=\widehat{G}^{T}+\widehat{Y}_{4}^{T}, \Pi_{34}=\widehat{Y}_{4}^{T} \\
& \Pi_{44}=-2 \widehat{T}, \Pi_{15}=-\widehat{Y}_{5}^{T}, \Pi_{25}=\Pi_{35}=\widehat{Y}_{5}^{T}, \Pi_{55}=-I \\
& \Pi_{16}=\widehat{P} \mathbb{A}^{T}, \Pi_{26}=\widehat{P} \mathbb{A}_{d}^{T}, \Pi_{46}=-\widehat{T} \mathbb{B}^{T}-F_{c}^{T} \mathbb{R}^{T} \\
& \Pi_{56}=\mathbb{B}_{w}^{T}, \Pi_{66}=-\widehat{P}, \Pi_{17}=\widehat{P} \mathbb{C}_{z}^{T}, \Pi_{77}=-\gamma I
\end{aligned}
$$

then, an anti-windup compensation $E_{c}=F_{c} \widehat{T}^{-1}$ exists, such that the closed-loop system (9) satisfies

(1) asymptotic stability;

(2) the performance index (10).

Proof 1: To prove this theorem, let us consider the following Lyapunov function

$$
\begin{aligned}
V(k)= & V_{1}(k)+V_{2}(k)+V_{3}(k) \\
= & \xi^{T}(k) P \xi(k)+\sum_{l=k-d(k)}^{k-1} \xi^{T}(l) Q \xi(l) \\
& +\sum_{l=-d_{M}+2}^{-d_{m}+1} \sum_{m=k+l-1}^{k-1} \xi^{T}(m) R \xi(m)
\end{aligned}
$$

and let us compute the difference of the Lyapunov function

$$
\begin{aligned}
\Delta V_{1}(k)= & \xi^{T}(k+1) P \xi(k+1)-\xi^{T}(k) P \xi(k) \\
\Delta V_{2}(k)= & \sum_{l=k+1-d(k+1)}^{k} \xi^{T}(l) Q \xi(l)-\sum_{l=k-d(k)}^{k-1} \xi^{T}(l) Q \xi(l) \\
= & \xi^{T}(k) Q \xi(k)-\xi^{T}(k-d(k)) Q \xi(k-d(k)) \\
& +\sum_{l=k+1-d_{m}}^{k-1} \xi^{T}(l) Q \xi(l)-\sum_{l=k+1-d(k)}^{k-1} \xi^{T}(l) Q \xi(l) \\
& +\sum_{l=k+1-d(k+1)}^{k-d_{m}} \xi^{T}(l) Q \xi(l) \\
\Delta V_{3}(k)= & \sum_{l=}^{-d_{m}+1}\left[d_{M+2}^{k} \xi_{m=k+l}^{T}(m) R \xi(m)\right. \\
& \left.-\sum_{m=k+l-1}^{k-1} \xi^{T}(m) R \xi(m)\right]_{l=k+1-d_{M}}^{k-d_{m}} \xi^{T}(l) R \xi(l) \\
= & d_{M m} \xi^{T}(k) R \xi(k)-\sum_{l 5)}
\end{aligned}
$$

where $d_{M m}=d_{M}-d_{m}$.

Since (5) with $\forall Q<R$, one can easily see that

$$
\begin{aligned}
& \sum_{l=k+1-d_{m}}^{k-1} \xi^{T}(l) Q \xi(l)-\sum_{l=k+1-d(k)}^{k-1} \xi^{T}(l) Q \xi(l) \\
& \leq \sum_{l=k+1-d_{m}}^{k-1} \xi^{T}(l) Q \xi(l)-\sum_{l=k+1-d_{m}}^{k-1} \xi^{T}(l) Q \xi(l)=0
\end{aligned}
$$

$$
\begin{aligned}
& \sum_{l=k+1-d(k+1)}^{k-d_{m}} \xi^{T}(l) Q \xi(l)-\sum_{l=k+1-d_{M}}^{k-d_{m}} \xi^{T}(l) R \xi(l) \\
& \quad \leq \sum_{l=k+1-d(k+1)}^{k-d_{m}} \xi^{T}(l) R \xi(l)-\sum_{l=k+1-d_{M}}^{k-d_{m}} \xi^{T}(l) R \xi(l) \\
& \quad \leq \sum_{l=k+1-d_{M}}^{k-d_{m}} \xi^{T}(l) R \xi(l)-\sum_{l=k+1-d_{M}}^{k-d_{m}} \xi^{T}(l) R \xi(l)=0
\end{aligned}
$$

From (13)-(17), it follows that

$$
\begin{aligned}
\Delta V(k)= & \xi^{T}(k+1) P \xi(k+1)+\xi^{T}(k)\left(-P+Q+d_{M m}\right. \\
& \times R) \xi(k)-\xi^{T}(k-d(k)) Q \xi(k-d(k))
\end{aligned}
$$

Using the Newton-Leibniz formula provides for any appropriately dimensioned matrices $Y_{1, \ldots, 5}$ yields

$$
\begin{aligned}
& {\left[\xi^{T}(k) Y_{1}+\xi^{T}(k-d(k)) Y_{2}+\sum_{i=k-d(k)}^{k-1} y^{T}(i) Y_{3}\right.} \\
& \left.+\psi^{T}(\mathbb{K} \xi(k-d(k))) Y_{4}+w^{T}(k) Y_{5}\right][-\xi(k) \\
& \left.+\xi(k-d(k))+\sum_{i=k-d(k)}^{k-1} y(i)\right]=0
\end{aligned}
$$


where $y(i)=\xi(i+1)-\xi(i)$.

On the other hand, according to the system equation (9), we have

$$
\begin{aligned}
\Delta \mathscr{V}(k) \leq & {\left[\mathbb{A} \xi(k)+\mathbb{A}_{d} \xi(k-d(k))-\left(\mathbb{B}+\mathbb{R} E_{c}\right)\right.} \\
& \left.\times \psi(\mathbb{K} \xi(k-d(k)))+\mathbb{B}_{w} w(k)\right]^{T} P[\mathbb{A} \xi(k) \\
& +\mathbb{A}_{d} \xi(k-d(k))-\left(\mathbb{B}+\mathbb{R} E_{c}\right) \psi(\mathbb{K} \xi(k-d(k))) \\
& \left.+\mathbb{B}_{w} w(k)\right]+\xi^{T}(k)\left[-P+Q+d_{M m} R\right] \xi(k) \\
& -\xi^{T}(k-d(k)) Q \xi(k-d(k))+2\left[\xi^{T}(k) Y_{1}\right. \\
& +\xi^{T}(k-d(k)) Y_{2}+\sum_{i=k-d(k)}^{k-1} y^{T}(i) Y_{3}+w^{T}(k) Y_{5} \\
& \left.+\psi^{T}(\mathbb{K} \xi(k-d(k))) Y_{4}\right][-\xi(k)+\xi(k-d(k)) \\
& \left.+\sum_{i=k-d(k)}^{k-1} y(i)\right]-2 \psi^{T}(\mathbb{K} \xi(k-d(k))) T \\
& \times[\psi(\mathbb{K} \xi(k-d(k)))-G \xi(k-d(k))] \\
& -w^{T}(k) w(k)+\frac{1}{\gamma} z^{T}(k) z(k)
\end{aligned}
$$

where $\Delta \mathscr{V}(k)=\Delta V(k)-w^{T}(k) w(k)+\frac{1}{\gamma} z^{T}(k) z(k)$. By simple manipulation, (20) can be rewritten as

$$
\Delta V(k)-w^{T}(k) w(k)+\frac{1}{\gamma} z^{T}(k) z(k) \leq \eta^{T}(k) \Psi \eta(k)
$$

where

$$
\begin{gathered}
\Psi=\left[\begin{array}{ccccc}
\Psi_{11} & \Psi_{12} & \Psi_{13} & \Psi_{14} & \Psi_{15} \\
* & \Psi_{22} & \Psi_{23} & \Psi_{24} & \Psi_{25} \\
* & * & \Psi_{33} & \Psi_{34} & \Psi_{35} \\
* & * & * & \Psi_{44} & \Psi_{45} \\
* & * & * & * & \Psi_{55}
\end{array}\right], \\
\eta^{T}(k)=\left[\begin{array}{cccc}
\xi^{T}(k) & \xi^{T}(k-d(k)) & \sum_{i=k-d(k)}^{k-1} y^{T}(i) \\
\psi^{T}(\mathbb{K} \xi(k-d(k))) & w^{T}(k)
\end{array}\right]
\end{gathered}
$$

and

$\Psi_{11}=\mathbb{A}^{T} P \mathbb{A}-P+Q+d_{M m} R-Y_{1}-Y_{1}^{T}+\frac{1}{\gamma} \mathbb{C}_{z}^{T} \mathbb{C}_{z}$

$\Psi_{12}=\mathbb{A}^{T} P \mathbb{A}_{d}+Y_{1}-Y_{2}^{T}, \Psi_{22}=\mathbb{A}_{d}^{T} P \mathbb{A}_{d}-Q+Y_{2}+Y_{2}^{T}$

$\Psi_{13}=Y_{1}-Y_{3}^{T}, \Psi_{23}=Y_{2}+Y_{3}^{T}, \Psi_{33}=Y_{3}+Y_{3}^{T}, \Psi_{34}=Y_{4}^{T}$

$\Psi_{14}=-\mathbb{A}^{T} P\left(\mathbb{B}+\mathbb{R} E_{c}\right)-Y_{4}^{T}, \Psi_{15}=\mathbb{A}^{T} P \mathbb{B}_{w}-Y_{5}^{T}$

$\Psi_{24}=-\mathbb{A}_{d}^{T} P\left(\mathbb{B}+\mathbb{R} E_{c}\right)+G^{T} T^{T}+Y_{4}^{T}, \Psi_{35}=Y_{5}^{T}$

$\Psi_{44}=\left(\mathbb{B}+\mathbb{R} E_{c}\right)^{T} P\left(\mathbb{B}+\mathbb{R} E_{c}\right)-2 T, \Psi_{25}=\mathbb{A}_{d}^{T} P \mathbb{B}_{w}+Y_{5}^{T}$

$\Psi_{45}=-\left(\mathbb{B}+\mathbb{R} E_{c}\right)^{T} P \mathbb{B}_{w}, \Psi_{55}=\mathbb{B}_{w}^{T} P \mathbb{B}_{w}-I$

The matrix $\Psi$ can be rewritten as $\Psi=\Upsilon+\Gamma=\Upsilon+L^{T} P L$ where

$\Upsilon=\left[\begin{array}{ccccc}\Upsilon_{11} & \Upsilon_{12} & \Upsilon_{13} & \Upsilon_{14} & \Upsilon_{15} \\ * & \Upsilon_{22} & \Upsilon_{23} & \Upsilon_{24} & \Upsilon_{25} \\ * & * & \Upsilon_{33} & \Upsilon_{34} & \Upsilon_{35} \\ * & * & * & \Upsilon_{44} & 0 \\ * & * & * & * & \Upsilon_{55}\end{array}\right], L=\left[\begin{array}{c}\mathbb{A}^{T} \\ \mathbb{A}_{d}^{T} \\ 0 \\ -\left(\mathbb{B}+\mathbb{R} E_{c}\right)^{T} \\ \mathbb{B}_{w}^{T}\end{array}\right]$ and

$$
\begin{aligned}
& \Upsilon_{11}=-P+Q+d_{M m} R-Y_{1}-Y_{1}^{T}+\frac{1}{\gamma} \mathbb{C}_{z}^{T} \mathbb{C}_{z} \\
& \Upsilon_{12}=Y_{1}-Y_{2}^{T}, \Upsilon_{22}=-Q+Y_{2}+Y_{2}^{T}, \Upsilon_{13}=Y_{1}-Y_{3}^{T} \\
& \Upsilon_{23}=Y_{2}+Y_{3}^{T}, \Upsilon_{33}=Y_{3}+Y_{3}^{T}, \Upsilon_{14}=-Y_{4}^{T} \\
& \Upsilon_{24}=G^{T} T^{T}+Y_{4}^{T}, \Upsilon_{34}=Y_{4}^{T}, \Upsilon_{44}=-2 T \\
& \Upsilon_{15}=-Y_{5}^{T}, \Upsilon_{25}=Y_{5}^{T}, \Upsilon_{35}=Y_{5}^{T}, \Upsilon_{55}=-I
\end{aligned}
$$

Then, we have

$$
\Delta V(k)-w^{T}(k) w(k)+\frac{1}{\gamma} z^{T}(k) z(k) \leq \eta^{T}(t)\left(\Upsilon+L^{T} P L\right) \eta(t)
$$

It is clear that if

$$
\Upsilon+L^{T} P L<0
$$

then

$$
\Delta V(k)-w^{T}(k) w(k)+\frac{1}{\gamma} z^{T}(k) z(k)<0
$$

Thus, the following condition is obtained by applying the Schur complement to (22)

$$
\left[\begin{array}{cccccc}
\Upsilon_{11} & \Upsilon_{12} & \Upsilon_{13} & \Upsilon_{14} & \Upsilon_{15} & \mathbb{A}^{T} P \\
* & \Upsilon_{22} & \Upsilon_{23} & \Upsilon_{24} & \Upsilon_{25} & \mathbb{A}_{d}^{T} P \\
* & * & \Upsilon_{33} & \Upsilon_{34} & \Upsilon_{35} & 0 \\
* & * & * & \Upsilon_{44} & 0 & -\left(\mathbb{B}+\mathbb{R}_{c}\right)^{T} P \\
* & * & * & * & \Upsilon_{55} & \mathbb{B}_{w}^{T} P \\
* & * & * & * & * & -P
\end{array}\right]<0
$$

Pre- and post-multiplying (24) by $\Delta=\operatorname{diag}\left\{P^{-1}, P^{-1}, P^{-1}\right.$, $\left.T^{-1}, I, P^{-1}\right\}$. Then, applying the Schur complement and taking the following changes of variables

$$
\begin{aligned}
& \widehat{P}=P^{-1}, \widehat{T}=T^{-1}, F_{c}=E_{c} \widehat{T}, \widehat{G}=G \widehat{P}, \widehat{Y}_{5}=Y_{5} \widehat{P} \\
& \widehat{\Lambda}=\widehat{P} \Lambda \widehat{P} \text { with } \Lambda=Q, R, Y_{1}, Y_{2}, Y_{3}, \text { and } \widehat{Y}_{4}=\widehat{T} Y_{4} \widehat{P}
\end{aligned}
$$

We obtain the condition (11). Since (11) holds, the condition (23) is satisfied. Now, summing up (23) from 0 to $\infty$ with respect to $\mathrm{k}$ yields,

$$
V(\infty)<V(0)+\sum_{k=0}^{\infty}\left(w^{T}(k) w(k)-\frac{1}{\gamma} z^{T}(k) z(k)\right)
$$

Under the zero initial condition $V(0)=0$ and by noting that $V(\infty) \geq 0$, we have (10) which implies that system (9) has its restricted $\mathscr{L}_{2}$-gain from $w(k)$ to $z(k)$ less than $\gamma$. Now taking $w(k)=0$, it is easy to see that $\Delta V(k)<0$. The proof is completed.

\section{B. Optimization Problem}

Verifying LMIs of Theorem 3.1 ensures that the closed loop system (9) presents bounded trajectories for any admissible perturbation. For a non-null positive bound on the $\mathscr{L}_{2}-$ norm of the admissible disturbances, the idea is to minimize the upper bound of the $\mathscr{L}_{2}$-gain of $w(k)$ on $z(k)$. Considering that the initial condition is null, this can be obtained from the solution of the following convex optimization problem

$$
\min \gamma \text { subject to (11) }
$$




\section{Implementation Constraints}

The control signal for the network is given by

$$
\delta p(k)=u(k)=y_{c}(k)=\left[\begin{array}{lll}
\mathbb{K}_{1} & \mathbb{K}_{2} & \mathbb{K}_{3}
\end{array}\right] \xi(k)
$$

In order to relax the computing resources to implement our proposal on a real network we use an approximation as follows

$$
\begin{aligned}
\delta W(k)=W(k)-W_{0} & =\frac{R T T}{N}\left(\frac{N W(k)}{R T T}-C_{0}\right) \\
& =\frac{R T T}{N}\left(\text { flow rate }-C_{0}\right) \\
& =\frac{R T T}{N} \times \text { rate of mismatch }(28)
\end{aligned}
$$

Furthermore, as in [4], one can also notice that the rate of mismatch is the rate at which the queue length grows when the buffer is nonempty. Therefore, we can approximate it by $\frac{\delta q}{T}$ where $\frac{1}{T}$ is the sampling frequency.

Hence, (27) becomes

$$
\begin{aligned}
\delta p(k) & =\left(\mathbb{K}_{1} \frac{R T T}{N T}+\mathbb{K}_{2}\right) \delta q(k)+\mathbb{K}_{3} x_{c}(k) \\
& =\left[\begin{array}{lll}
0 & \mathbb{K}_{1} \frac{R T T}{N T}+\mathbb{K}_{2} & \mathbb{K}_{3}
\end{array}\right] \xi(k)
\end{aligned}
$$

As pointed out before, to implement our AQM controller using (11), we first discretize (3).

\section{ILLUSTRATIVE EXAMPLE}

The above AQM controller is obtained via Theorem 3.1 using the LMI-toolbox of MATLAB. In order to demonstrate the effectiveness and applicability of proposed design methodology, some Matlab simulations of experiments are provided to compare the proposed design approach with RED control scheme for AQM routers. A single bottlenecked router running AQM controller (29) is considered in the simulations. In addition to the TCP flows addressed in the model, we also introduced FTP flows into the router to generate a realistic traffic scenario.

The round trip time is $R T T=0.253$, the bottleneck link capacity is $C_{0}=3750$, the operating point is $q_{0}=175$, the number of connections $N=60$. From the following model builder $W_{0}=\frac{R T T C_{0}}{N}, p_{0}=\frac{2}{W_{0}^{2}}$ and $T_{p}=R T T-\frac{q_{0}}{C_{0}}$ we can calculate the steady state discard probability, the propagation delay and the round trip time, respectively. For this setup, we assume $u_{0}=p_{0}$. On the other hand, bellow the matrices of output stabilizing controller are given explicitly

$A_{c}=0, B_{c}=1, C_{c}=8.4969 \times 10^{-6}, D_{c}=1.6996 \times 10^{-5}$

The simulation results are evaluated according to the disturbance signal which is defined as

$$
w(t)=\left\{\begin{array}{cc}
10, & 0 \leq t \leq 1 \\
0, & t \geq 1
\end{array}\right.
$$

Then, in order to compare the results of the proposed $\mathrm{AQM}$ controller with other AQM router design schemes, we introduce RED controller in the simulations.

From [12] a transfer function model for RED is $C(s)=$ $\frac{K \times L_{\text {red }}}{s+K}$ where $L_{r e d}=\frac{P_{\max }}{\max _{t h}-\min _{t h}}$. The RED parameters are chosen as that $K=0.005^{\text {th }}$ and the dynamic range $\left(\min _{t h}, \max _{t h}\right)$ are $(150,700)$ packets. REDs averaging weight $\alpha=1.33 \times 10^{6}$ and $P_{\max }=0.1$.

Fig. 2 and 3 depict the queue length and discard probability, respectively, of both AQM controller and RED with initial values $\xi_{0}=\left[\begin{array}{ll}0.5 & 0.5\end{array}\right]^{T}$ and the obtained anti-windup compensator from Theorem $3.1, E_{c}=-2.7461 \times 10^{-5}$ where $d_{M m}=0.246$ and $T=0.0001$. The resolution of the optimization problem (26) let us obtain $\sqrt{\gamma}=0.1809$.



Fig. 2. Variation of a queue over average value

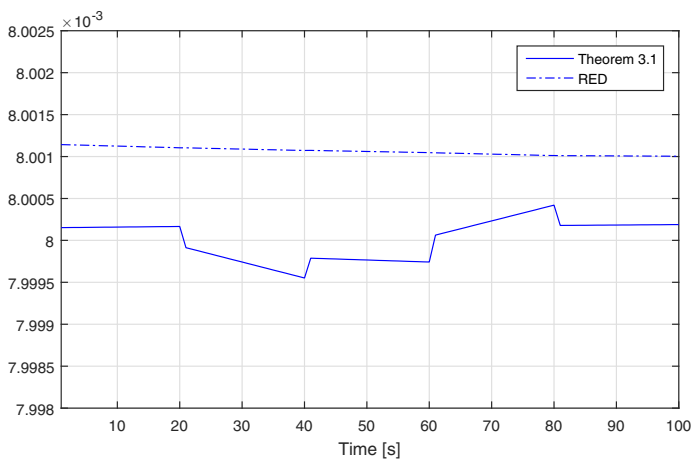

Fig. 3. Variation of discard probability over average value

It can be seen from Fig. 2 that all queue length were stabilized at target value when our AQM controller is used. Also, the proposed method has achieved superior performance with less probability of packet drop compared to RED as shown in Fig. 3. This proves that the compensator used can force the system to rapidly achieve the desired reference value. In addition, the achieved controller tackles one of the great weakness of previous AQM mechanisms as it does not require to be tuned for different operating conditions. Specifying the desired performance objective through the desired reference value, the proposed controller can also meet the other performance objectives.

\section{CONCLUSION}

It is clear that the dynamic anti-windup compensator reduces the probability dropping packet. Therefore, it increases the throughput of the networks of the users. A control theory approach has been successfully developed to solve congestion problem in TCP/IP Routers. The methodology has been validated by a numerical example, showing the improvements with respect to previous approaches in the literature.

The paper can be improved by using a more realistic model 
of Internet traffic within a large parameter set. A typical traffic mix can be used for the evaluation. More complex network topologies can be add. These may include the reverse-dumbbell topology with multiple congested gateways and realistic Internet-like topologies such as power-laws.

\section{ACKNOWLEDGMENT}

Funded by MiCInn DPI2014-54530-R and FEDER funds.

\section{REFERENCES}

[1] Alvarez, T., Design of PID controllers for TCP/AQM wireless networks, In Proceedings of the World Congress on Engineering, 2, pp. 1-8, 2012.

[2] Alvarez, Teresa, and Martinez, D., Handling the congestion control problem of TCP/AQM wireless networks with PID controllers, IAENG Transactions on Engineering Technologies. Springer Netherlands, pp. 365-379, 2013.

[3] Aoul, Y. H., Nafaa, A., Negru, D., and Mehaoua, A., FAFC: fast adaptive fuzzy AQM controller for TCP/IP networks, Proceedings of the Global Telecommunications Conference (GLOBECOM'04), 3, pp. 1319-1323, 2004.

[4] Azadegan, M., Beheshti, M. T. H., Tavassoli, B., Design of state feedback controller based on state-dependent delay modeling for congestion control in internet, Proceedings of the American Control Conference, pp. 2728-2732, 2013.

[5] Binotti, V., and Bender, F. A., Control theory based AQM for high speed networks, International Journal of Innovative Computing, Information and Control, 10, 6, pp. 2025-2033, 2014.

[6] Cho, H. C., Fadali, M. S., Lee, H., Neural network control for TCP network congestion, Proceedings of the American Control Conference, 5, pp. 3480-3485, 2005.

[7] El Fezazi, N., El Haoussi, F., Tissir, E. H., and Tadeo, F., Delay dependent anti-windup synthesis for time-varying delay systems with saturating actuators, International Journal of Computer Applications (0975 8887), 111, 1, pp. 1-6, 2015.

[8] El Fezazi, N., Tissir, E. H., and El Haoussi, F., Delay-dependent antiwindup design to address challenges in AQM congestion control for TCP/IP networks, Proceedings of the International Conference Advanced Information Technology, Services and Systems (AIT2S), Settat, Morocco, pp. 309-313, 2015.

[9] El Fezazi, N., Lamrabet, O., El Haoussi, F., Tissir, E. H., Alvarez, T., and Tadeo, F., Robust controller design for congestion control in TCP/IP routers, Proceedings of the International Conference on Systems and Control (ICSC), Marrakesh, Morocco, pp. 243-249,2016.

[10] El Fezazi, N., El Haoussi, F., Tissir, E. H., Husain, A. R., and Zakaria, M. I., Delay-dependent robust anti-windup synthesis approach to AQM in TCP/IP networks, Proceedings of the International Conference on Electrical and Information Technologies (ICEIT), Tanger, Morocco, pp. 294-299, 2016.

[11] Fengyuan, R., Yong, R., Xiuming, S., Design of a fuzzy controller for active queue management, Computer Communications, 25, 9, pp. 874-883, 2002.

[12] Floyd, S. and Jacobson, V., Random early detection gateways for congestion avoidance', IEEE/ACM Transactions on Networking, 1, 4, pp. 397-413, 1993.

[13] Hollot, C.V., Misra, V., Towsley, D., and Gong, W.-B., A control theoretic analysis of RED, IEEE INFOCOM 2001, pp. 1510-1519, 2001.

[14] Hollot, C.V., Misra, V., Towsley, D., and Gong, W.-B., On designing improved controllers for AQM routers supporting TCP flows, IEEE INFOCOM 2001, pp. 1726-1734, 2001

[15] Kahe, G., Jahangir, A. H., Ebrahimi, B., AQM controller design for TCP networks based on a new control strategy, Telecommunication Systems, 57, 4, pp. 295-311, 2014.

[16] Tarbouriech, S., Turner, M., Anti-windup design: an overview of some recent advances and open problems, Control Theory and Applications, IET, 3, 1, pp. 1-19, 2009.
[17] Yaghmaee Moghaddam, M. H., A fuzzy Active Queue Management mechanism for Internet congestion control, Proceedings of 3rd International Workshop on Advanced Computational Intelligence (IWACI), 25, 9, pp. 203-208, 2010.

[18] Yaghoubi Waskasi, M., Yazdanpanah, M. J., Yazdani, N., A new active queue management algorithm based on neural networks PI, Proceedings of 16th IFAC Triennial World Congress, pp. 1-6, 2005.

[19] Zhang, X. Q., Zhao, J., $L_{2}$-gain analysis and anti-windup design of discrete-time switched systems with actuator saturation, International Journal of Automation and Computing, 9, 4, pp. 369-377, 2012. 